\title{
EDUCACIÓN
}

\section{Desarrollo de la cultura científica en la formación inicial del docente}

\author{
Lilian Silvana Rodríguez Vera
}

\begin{abstract}
Resumen
Introducción: Hoy en día el debate se centra en todo lo relacionado a la formación docente, sobre las competencias requeridas para llevar a cabo dicha profesión. Indagar sobre las prácticas pedagógicas posibilita visualizar el estado del arte de la educación de un país, en este sentido, focalizar la mirada en los espacios educativos permite descubrir algunas pistas sobre lo que acontece en la realidad. Desde esa mirada, parece inevitable instalar el diálogo sobre cómo se desarrolla la cultura científica desde el trayecto formativo del docente.

En este estudio se aborda el análisis del rol del docente formador para el desarrollo de la cultura científica, desde la alfabetización científica y la representación social, en cómo la ciencia es concebida para su desarrollo.

Conviene señalar que el docente como mediador en la construcción de conocimientos tiene el gran desafío de desarrollar habilidades que posibiliten una mejor calidad de vida, en este sentido, se piensa que la ciencia contribuye; por lo que la clave en los procesos de enseñanza y aprendizaje sería el desarrollo de la cultura científica.
\end{abstract}

Objetivo: Analizar el rol del docente formador en el desarrollo de la cultura científica del Instituto Superior de Educación "Dr. Raúl Peña".

Material y Método: Esta investigación, de corte cualitativo, utiliza la metodología propuesta por el Manual de Ciencias Sociales de Quivy y Van Campenhoudt (2005), se enmarcó en la modalidad Investigación Acción y tiene un alcance exploratorio. Los sujetos participantes son los docentes del ISE. Las técnicas utilizadas fueron: encuesta, entrevista a profundidad y taller concientizador.

Resultados: Al decir de los participantes las prácticas pedagógicas desarrolladas en el ISE se enmarcan dentro del modelo tradicional de enseñanza y aprendizaje; aún son incipientes los espacios de discusión sobre cultura científica y conviene pensar en cómo sistematizar los informes elaborados en el marco de la

\footnotetext{
1. Instituto Superior de Educación "Dr. Raúl Peña". Dpto. Técnico Pedagógico-Dirección Académica.

Esta tesis se enmarca dentro del requisito de titulación del programa de maestría en "Investigación Científica con énfasis en métodos cualitativos y cuantitativos", financiada por el Consejo Nacional de Ciencia y Tecnología - CONACYT, a través del Programa Paraguayo para el Desarrollo de la Ciencia y Tecnología - PROCIENCIA con recursos del Fondo para la Excelencia de la Educación e Investigación - FEEI.

E-mail: silvanarodriguez17@gmail.com

DOI: $10.26885 /$ rcei.foro.2018.70
} 
asignatura Práctica Profesional ya que al parecer esos saberes precisan de más reflexión y sistematización; urge pensar en un modelo de gestión que posibilite desarrollar la cultura científica.

Conclusiones: En relación al primer objetivo específico los hallazgos señalan que los docentes del ISE caracterizan la ciencia desde la mirada positivista, o sea, la ciencia busca permanentemente la certeza de los fenómenos.

En relación al segundo objetivo se pudo observar que al parecer los procesos de enseñanza y aprendizaje del ISE aún se fundamentan en un modelo pedagógico tradicional; en ese sentido señalaron que la curiosidad queda desvinculada del quehacer educativo.

En relación al último objetico se encontró que existen intenciones de potenciar el carácter científico de la práctica docente. Por otro lado, se ha mencionado la urgencia de mejorar la gestión directiva de los diferentes estamentos, ya que se percibe una escasa administración de las actividades a realizar, éstas son vistas como desligadas unas de otras.

Por lo que finalmente se puede señalar que el rol de los docentes formadores para la formación de hábitos científicos, desde las prácticas pedagógicas, representa una cuestión clave que necesita ser abordada desde espacios de diálogo, ya que la formación científica se encuentra en una etapa incipiente.

Palabras clave: ciencia, cultura científica, rol docente.

\section{RefEREnCias}

Fornet-Betancourt, R. (2006). La interculturalidad a prueba (Vol. 43). Mainz.

Quivy, R. \& Van Carnpenhoudt, L. (2005). Manual de Investigación en Ciencias Sociales. México DF: Limusa.

Rivarosa, A. S. \& Astudillo, C. S. (2013). Las prácticas científicas y la cultura: una reflexión necesaria para un educador de ciencias. Revista CTS, 45-66.

Stronquist, N. (2017). Investigación-acción: un nuevo enfoque sociológico. Revista Colombiana de Educación, 1-11.

Valladares, L. (2011). Hacia una educación científica comprehensiva e intercultural: las espirales de enseñanza-aprendizaje de la ciencia. Horizontes Educacionales, 31-48. 